\title{
Cytarabine-Thioguanine Regimen
}

National Cancer Institute

\section{Source}

National Cancer Institute. Cytarabine-Thioguanine Regimen. NCI Thesaurus. Code C63660.

A reg imen consisting of cytarabine and thioguanine used as post remission maintenance treatment for acute myeloid leukemia. Was orig inally used as an induction regimen, but has been widely replaced with anthracycline-containing regimens. 